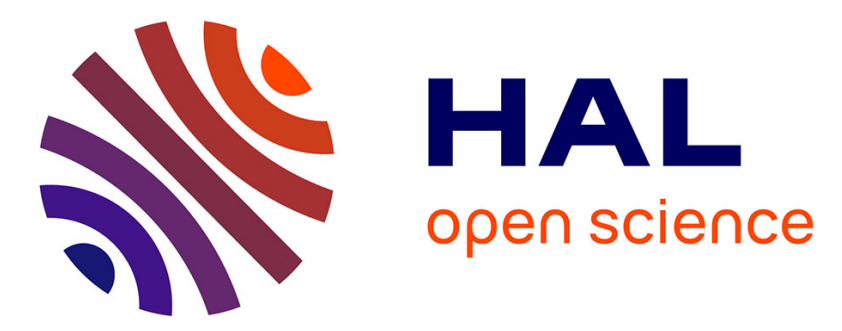

\title{
Supramolecular Assembly of TPE-Based Glycoclusters with Dicyanomethylene-4 H -pyran (DM) Fluorescent Probes Improve Their Properties for Peroxynitrite Sensing and Cell Imaging
}

Lei Dong, Mengqi Fu, Lifang Liu, Hai-hao Han, Yi Zang, Guo-rong Chen, Jia Li, Xiao-peng He, Sébastien Vidal

\section{To cite this version:}

Lei Dong, Mengqi Fu, Lifang Liu, Hai-hao Han, Yi Zang, et al.. Supramolecular Assembly of TPEBased Glycoclusters with Dicyanomethylene-4 H -pyran (DM) Fluorescent Probes Improve Their Properties for Peroxynitrite Sensing and Cell Imaging. Chemistry - A European Journal, 2020, 26 (63), pp.14445-14452. 10.1002/chem.202002772 . hal-03309703

\section{HAL Id: hal-03309703 https://hal.science/hal-03309703}

Submitted on 29 Oct 2021

HAL is a multi-disciplinary open access archive for the deposit and dissemination of scientific research documents, whether they are published or not. The documents may come from teaching and research institutions in France or abroad, or from public or private research centers.
L'archive ouverte pluridisciplinaire HAL, est destinée au dépôt et à la diffusion de documents scientifiques de niveau recherche, publiés ou non, émanant des établissements d'enseignement et de recherche français ou étrangers, des laboratoires publics ou privés. 


\title{
Supramolecular Assembly of TPE-Based Glycoclusters with Dicyanomethylene-4H-pyran (DM) Fluorescent Probes Improve Their Properties for Peroxynitrite Sensing and Cell Imaging
}

\author{
Lei Dong, ${ }^{[a, b]}$ Mengqi Fu, ${ }^{[a]}$ Lifang Liu, ${ }_{1}^{[a]}$ Hai-Hao Han, ${ }^{[a]}{ }^{2}$ i Zang, ${ }^{[c]}$ Guo-Rong Chen, ${ }^{[a]}$ Jia Li, ${ }^{*[c]}$ \\ Xiao-Peng $\mathrm{He}^{*[a]}$ and Sébastien Vidal*[b]
}

Abstract: Two red-emitting dicyanomethylene-4H-pyran (DM) based fluorescent probes were designed and used for peroxynitrite $\left(\mathrm{ONOO}^{-}\right)$detection. Nevertheless, the aggregation-caused quenching effect diminished the fluorescence and restricted their further applications. To overcome this problem, tetraphenylethylene (TPE) based glycoclusters were used to self-assemble with these DM probes to obtain supramolecular water-soluble glyco-dots. This self-assembly strat- egy enhanced the fluorescence intensity, leading to an enhanced selectivity and activity of the resulting glyco-dot comparing to DM probes alone in PBS buffer. The glyco-dots also exhibited better results during fluorescence sensing of intracellular $\mathrm{ONOO}^{-}$than the probes alone, thereby offering scope for the development of other similar supramolecular glyco-systems for chemical biological studies.

\section{Introduction}

Peroxynitrite $\left(\mathrm{ONOO}^{-}\right)$is an important reactive oxidant and nitration agent in physiological and pathological processes. It has been demonstrated to cause neuronal death in vivo such as brain ischemia, amyotrophic lateral sclerosis, Alzheimer's disease and Parkinson's disease. ${ }^{[1]}$ Peroxynitrite can damage tissues by releasing hydroxyl radical $\left({ }^{\circ} \mathrm{OH}\right)$ and nitrogen dioxide $\left(\mathrm{NO}_{2}{ }^{-}\right)$. Also, due to its efficient nitration and oxidation properties, biomolecules such as DNA, proteins, thiols and lipids are easily damaged triggering cancer, septic shock, atherosclerosis and multiple sclerosis. ${ }^{[2]}$ However, peroxynitrite is difficult to

[a] Dr. L. Dong, M. Fu, Prof. L. Liu, H.-H. Han, Prof. G.-R. Chen, Prof. X.-P. He Key Laboratory for Advanced Materials and Joint International Research Laboratory of Precision Chemistry and Molecular Engineering Feringa Nobel Prize Scientist Joint Research Center School of Chemistry and Molecular Engineering Frontiers Center for Materiobiology and Dynamic Chemistry East China University of Science and Technology 130 Meilong Rd., Shanghai 200237 (P. R. China) E-mail:xphe@ecust.edu.cn

[b] Dr. L. Dong, Dr. S. Vidal

Institut de Chimie et Biochimie Moléculaires et Supramoléculaires Laboratoire de Chimie Organique 2-Glycochimie, UMR 5246, CNRS Université Claude Bernard Lyon 1, Université de Lyon 1, Rue Victor Grignard, 69622 Villeurbanne (France) E-mail: sebastien.vidal@univ-lyon1.fr

[c] Prof. Y. Zang, J. Li

National Center for Drug Screening, State Key Laboratory of Drug Research Shanghai Institute of Materia Medica, Chinese Academy of Sciences 189, Guo Shoujing Rd., Shanghai 201203 (P. R. China) E-mail:jli@simm.ac.cn

$\square$ Supporting information and the ORCID identification number(s) for the

(iD author(s) of this article can be found under: https://doi.org/10.1002/chem.202002772. detect in biological systems due to its short half-life $(\approx 10$ $20 \mathrm{~ms}){ }^{[2]}$ Despite this short lifetime, the level of intracellular peroxynitrite is a key indicator to monitor and predict diseases effectively.

Over the past decade, fluorescent probes for peroxynitrite detection and imaging in cells with different mechanisms were reported frequently. These probes displayed sensitivity, selectivity, high spatial and temporal resolution for peroxynitrite detection in vivo. ${ }^{[3]}$ For the interference from background autofluorescence, the development of long-wavelength fluorescent probes was required to detect peroxynitrite in tissues. Sikora et al. reported the reactivity of peroxynitrite with aromatic boronates was better than hypochlorous acid $\left(\mathrm{HOCl} / \mathrm{ClO}^{-}\right)$and hydrogen peroxide $\left(\mathrm{H}_{2} \mathrm{O}_{2}\right) \cdot{ }^{[4]}$ Since then, various boronate fluorescent probes were reported. ${ }^{[5-7]}$ Besides, self-assembled nanostructures in relation to therapeutic strategies for neurodegenerative disorders were reported. ${ }^{[8]}$

In our previous studies, we developed fluorescent glycoprobes based on organic dyes modified with carbohydrate moieties to enhance not only their water solubility but also for targeting cancer cells that over-express specific carbohydrate receptors. These glycoprobes achieved the sensitive detection and targeted imaging of ions or biomolecules. ${ }^{[9]}$ Dicyanomethylene-4H-pyran (DM), due to its broad absorption band and relatively long emission wavelength, was widely used for biosensing and bioimaging. ${ }^{[10]}$ However, the fluorescence properties of DM-based probes were generally influenced by the aggregation-caused quenching (ACQ) effect from the low water-solubility in PBS buffer thus restricting the sensitivity and further biological applications. ${ }^{[11]}$ Glycoclusters are watersoluble and typically display high affinity for cell surface receptors and have been thus used for targeted cell imaging and 
drug delivery. ${ }^{[12]}$ Recently, through the self-assembly between perylenediimide (PDI)-based galactoclusters and a DM dye, we have developed a water-soluble supramolecular "glyco-dot" system for the targeted fluorescence imaging of liver cancer cells that over-express galactose receptors. ${ }^{[13]}$ However, in that study DM was only used as an imaging agent rather than a chemical probe for sensing intracellular signaling molecules.

Tetraphenylethene (TPE) is a fluorogen with aggregation-induced emission properties, and TPE-based glycoclusters have been developed for lectin sensing. ${ }^{[14]}$ The amphiphilic TPEbased glycoclusters in PBS buffer, might well self-assemble with the hydrophobic DM probes. The corresponding glycodots with satisfying water solubility were monitored for their response peroxynitrite in PBS buffer. Therefore, we have designed two DM-boronate fluorescent probes (DMB1 and DMB2) for peroxynitrite detection. Their molecular design with two different moieties between the DM core and the phenylboronic acid will be used to determine the influence on the fluorescence properties. The resulting probes could release strong fluorescence emission upon exposure to peroxynitrite in PBS buffer mixed with $20 \% \mathrm{MeCN}$, but exhibited low fluorescence changes due to the ACQ effect in pure PBS buffer. To overcome this problem, TPE-based glycoclusters bearing two or four carbohydrate residues (TPE-Gly ${ }_{2}$ and TPE-Gly T $_{4}$ were designed and synthesized through CuAAC cycloaddition to improve the water solubility of the DM probes. The DM-probes could readily self-assemble in the hydrophobic core of the TPE-based glycoclusters to obtain the supramolecular glycodots system with fluorescence enhancement or FRET phenomena (Scheme 1). The water-soluble glyco-dots were therefore investigated for their response to peroxynitrite in terms of sensitivity, efficiency and selectivity in comparison to the DM probes alone in pure PBS buffer. These glyco-dots were also studied for their imaging properties of exogenous and endogenous peroxynitrite in living cells.

\section{Results and Discussion}

\section{Synthesis of DM-based fluorescent probes}

For their excellent fluorescence properties, we have introduced boronic moieties on the DM fluorogen for efficient peroxynitrite detection. We conjugated different phenylboronates moieties with the known 4-(dicyanomethylene)-2-(tert-butyl)-6methyl-4H-pyran $5^{[15]}$ (Scheme 2). DMB1 was obtained by condensation of 4-formylphenylboronic acid pinacol ester with compound $\mathbf{5}$ in the presence of catalytic piperidine. The electron-poor boronate ester could block the ICT effect to quench fluorescence. DM-OH, having strong fluorescence emission, was synthesized similarly from compound $\mathbf{5}$ and 4-hydroxybenzaldehyde. Further functionalization with a 4-bromomethylbenzeneboronic acid pinacol ester provided probe DMB2. The intramolecular $D-\pi-A$ system was influenced by the PET effect and thereby quenched the fluorescence.

Meanwhile, several TPE-based glycoclusters were synthesized. Dimethoxy-TPE ${ }^{[16]} \mathbf{2}$ was demethylated then propargylated and conjugated with azido-functionalized glycosides ( $\beta$-gal-

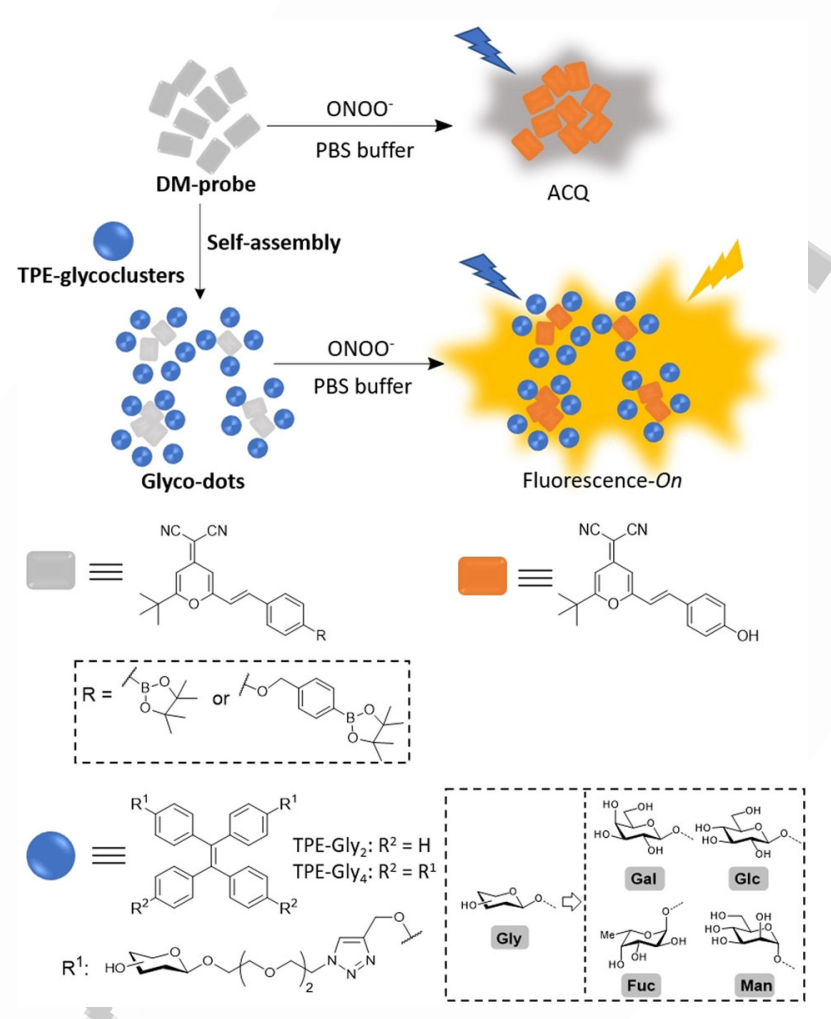

Scheme 1. Self-assembly process of glyco-dots for peroxynitrite detection and structures of DMB1, DMB2, DM-OH and TPE-based glycoclusters.

actose, $\beta$-glucose, $\alpha$-mannose and $\alpha$-fucose) through azidealkyne click chemistry (Scheme 2). After deprotection under $\mathrm{MeOH} / \mathrm{H}_{2} \mathrm{O} / \mathrm{Et}_{3} \mathrm{~N}$ solution, divalent TPE-based glycoclusters (TPE-Gly ${ }_{2}$ ) were obtained, while the tetravalent TPE-based glycoclusters (TPE-Gly ${ }_{4}$ ) were previously reported by us. ${ }^{[14]}$

\section{Fluorescence properties of the DM probes}

We initially investigated the UV/Vis and fluorescence properties of DMB1 and DMB2 in PBS buffer mixed with $20 \% \mathrm{MeCN}$ to improve the solubility (Figure S1). The two probes had wide absorption areas (300-450 $\mathrm{nm}$ ) originally without the addition of peroxynitrite. Compared with the low fluorescence intensity of DMB2 $(\Phi<0.01)$ influenced by PET effect, the fluorescence of DMB1 $(\Phi<0.01)$ was quenched completely by the electronpoor boronate blocking the ICT effect. In the presence of peroxynitrite $(100 \mu \mathrm{M})$, two novel absorption peaks were observed at $320 \mathrm{~nm}$ and $470 \mathrm{~nm}$, along with a colorimetric response from pale yellow to orange (Figure S2). Meanwhile, an obvious fluorescence enhancement was observed at $625 \mathrm{~nm}$, which displayed a large Stokes shift $(145 \mathrm{~nm})$. The fluorescence intensities of the solutions containing DMB1 $(\Phi=0.11)$ and DMB2 $(\Phi=0.10)$ increased after addition of peroxynitrite based on the fluorescence properties of DM-OH (Table S1).

Plausible sensing mechanisms of the two DM probes for peroxynitrite can be proposed. For probe DMB1, the electronpoor boron atom was electrophilic enough to allow condensation with the anionic and nucleophilic oxygen atom on peroxynitrite. Rearrangement to the borate with loss of nitrite, cleav- 


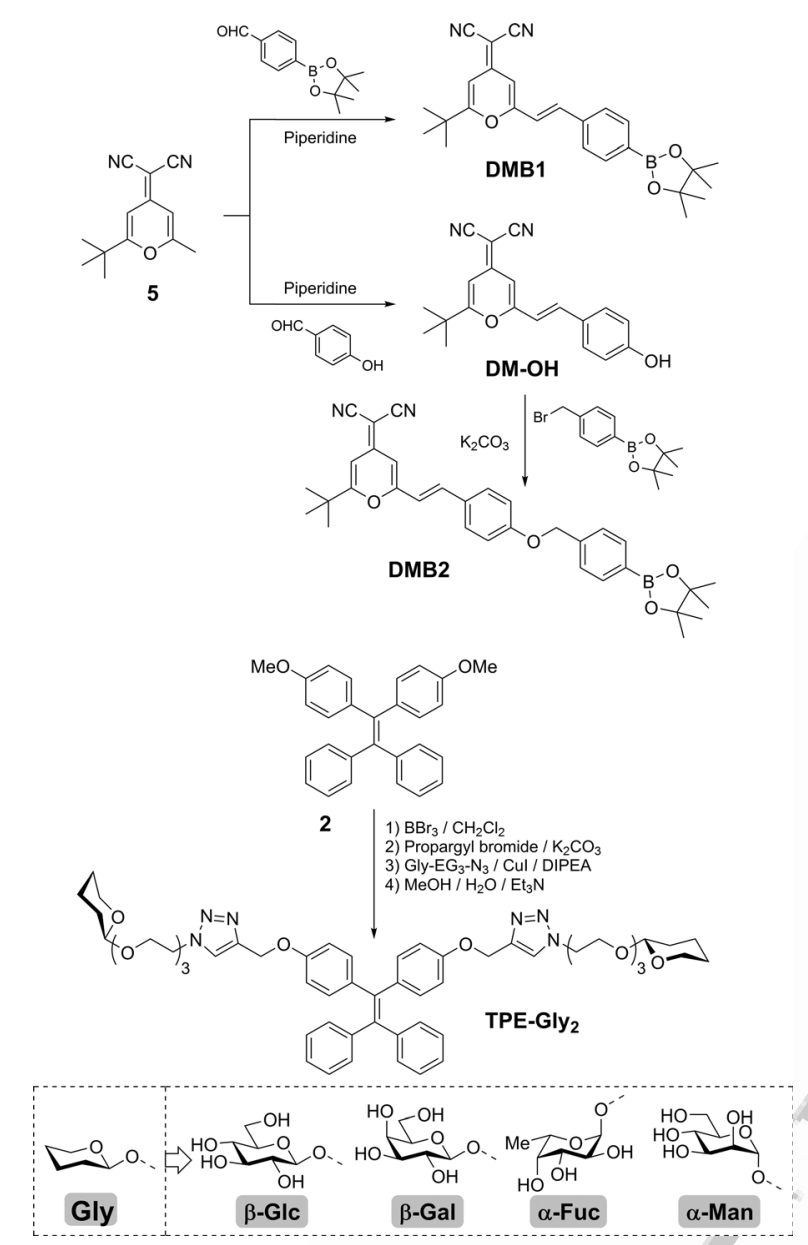

Scheme 2. Synthesis of DM-based fluorescent probes (DMB1, DMB2 and $\mathrm{DM}-\mathrm{OH}$ ) and the divalent TPE-based glycoclusters (TPE-Gly $)_{2}$. See Supporting Information for details.

age of the $\mathrm{C}-\mathrm{B}$ bond and creation of the $\mathrm{C}-\mathrm{O}$ bond, and further hydrolysis afforded the desired DM-OH with strong fluorescence emission, followed by the ICT effect recovery (Scheme $\mathrm{S} 1 \mathrm{~A})$. The response process of DMB2 would include one additional rearrangement step in comparison to DMB1, which is the spontaneous elimination of quinone methide to afford the desired phenol DM-OH (Scheme S1B). This two-step response mechanism would probably influence the chemical reactivity and fluorescence intensity of DMB2 after responding to peroxynitrite in vitro or in vivo. Mass spectrometry (HRMS) and chromatography (HPLC) analyses further confirmed the response mechanism for both DM probes in PBS buffer with $20 \% \mathrm{MeCN}$ (Figure 1). Both probes incubated with peroxynitrite yielded the same new compound (DM-OH, $m / z$ 339.1, Figure S3). Also, the HPLC analysis of the starting materials and incubated probes clearly indicated the transformation of both DMB1 (14.1 $\mathrm{min}$ ) and DMB2 (15.3 $\mathrm{min}$ ) into the fluorescent DM-OH (10.5 min).

However, the presence of a large amount of MeCN (20\%) in PBS is toxic to cells, thus limiting the biological application of the probes. Indeed, we determined that DMB1 and DMB2 when reacted with peroxynitrite also suffered from ACQ effect in full PBS buffer (Figure S4). To overcome this problem, we

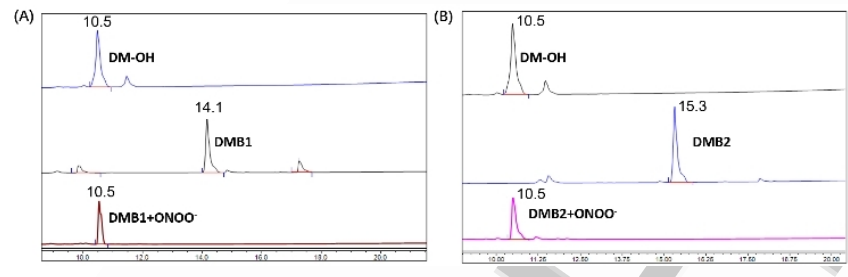

Figure 1. HPLC analysis for the response mechanism of (A) DMB1 $(10 \mu \mathrm{M})$ and (B) DMB2 (10 $\mu \mathrm{M})$ in PBS buffer (pH 7.4, $0.01 \mathrm{M})$ with $20 \% \mathrm{MeCN}$.

sought to use our previously developed TPE-based glycoclusters (TPE-Gly ${ }_{2}$ and TPE-Gly ${ }_{4}$ ) to self-assemble with the DM probes in order to enhance their water dispersibility.

\section{Glyco-dots self-assembly from DM-based probes and TPE- based glycoclusters}

To improve the water solubility of the DM fluorescent dye, we investigated its self-assembly with TPE-glycoclusters in PBS buffer. The hydrophobic cavity of TPE-glycoclusters may accommodate DM probes inside to avoid the ACQ restriction. We employed DLS and TEM to measure the size and morphology of the resulting glyco-dots. Next, the fluorescence variation of glyco-dots were measured at $365 \mathrm{~nm}$ or $470 \mathrm{~nm}$ excitation under different concentration conditions.

Dynamic light scattering (DLS) and transmission electron microscope (TEM) were used to measure the size distribution and morphology, respectively, of DMB1, DMB2, TPE-Gal ${ }_{2}$, TPE-Gal ${ }_{4}$ and the corresponding glyco-dots. We observed that the size of TPE$-G a l_{4}(\approx 60 \mathrm{~nm})$ was larger than that of TPE-Gal ${ }_{2}($ $\approx 20 \mathrm{~nm}$ ) by DLS (Figure 2). This suggests that the tetra-substitution pattern of TPE represents a larger molecular volume than the disubstituted system in their aggregated status. The diameter of DMB2 $(\approx 90 \mathrm{~nm})$ was determined to be larger than that of DMB1 $(\approx 20 \mathrm{~nm})$, which illustrated that DMB2 had a lower water solubility leading to aggregation in PBS solution. After incubating DM probes with TPE-glycoclusters, the size distribution of the supramolecular glyco-dots was nar-
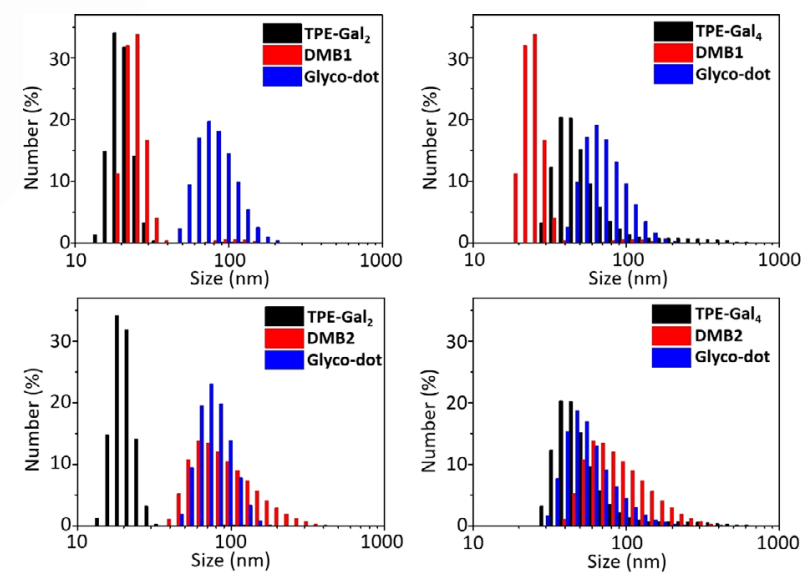

Figure 2. Aggregate size variation of DM probes, TPE-based glycoclusters and their glyco-dots after self-assembly obtained by DLS. 
rowed comparing to the DM probes alone, suggesting the complete self-assembly between the two partners (Figure 2).

In their representative TEM images (Figure 3), sheet-like and filament-like structures were observed for DMB1 and DMB2, respectively. The TPE-Gal ${ }_{4}$ aggregates appeared to be larger than those of TPE-Gal ${ }_{2}$, which is in agreement with the DLS results. Interestingly, after self-assembly, structurally uniform glyco-dots formed between the TPE glycoclusters and DM probes (Figure 6). The DLS and TEM data well corroborated that the DM probes could self-assemble into the hydrophobic core of TPE-glycoclusters to construct the well-dispersible supramolecular glyco-dots in aqueous solution.

Subsequently, DM probes $(10 \mu \mathrm{M})$ and TPE-glycoclusters $(150 \mu \mathrm{M})$ were incubated in PBS buffer $(\mathrm{pH} 7.4,0.01 \mathrm{M})$ leading to supramolecular glyco-dots. The fluorescence of TPE $\left(\lambda_{\mathrm{em}}=\right.$ $490 \mathrm{~nm}$ ) was gradually decreased with an increasing concentration of DM probes $\left(\lambda_{\mathrm{ex}}=470 \mathrm{~nm}\right)$ as excited at $320 \mathrm{~nm}$ (Figure 4). This implies a Förster resonance energy transfer (FRET) between the two closely associated fluorophores. Meanwhile, the fluorescence intensity of DMB2 (acceptor) was increased $\left(\lambda_{\mathrm{em}}=625 \mathrm{~nm}\right)$ with TPE-glycoclusters (donor), which agrees with a typical FRET process $\left(\Phi_{\mathrm{ET}}=68 \%\right.$ from TPE-Gal ${ }_{2}$, $\Phi_{\mathrm{ET}}=75 \%$ from TPE-Gal ${ }_{4}$ ). Nevertheless, the fluorescence in-
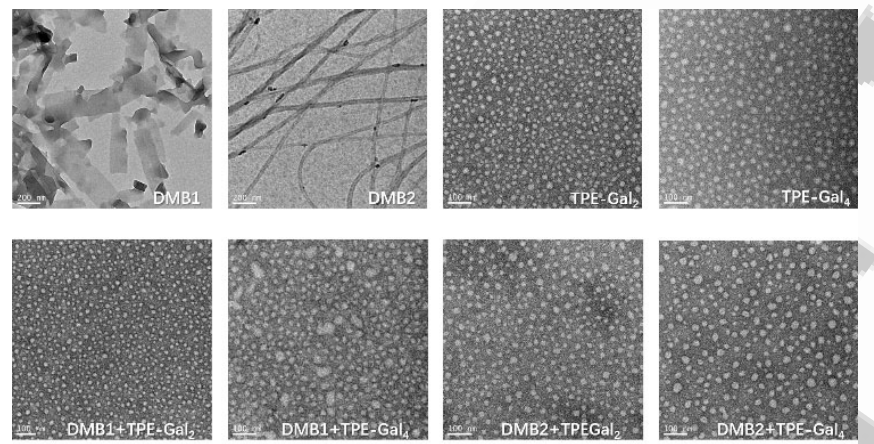

Figure 3. Aggregation of DM probes, TPE-based glycoclusters and their glyco-dots after self-assembly obtained by TEM.
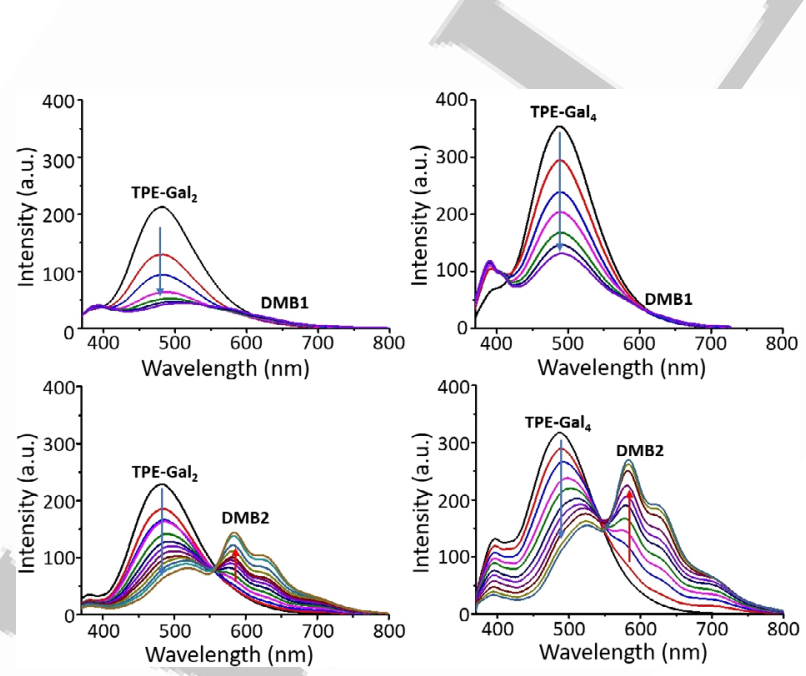

Figure 4. Fluorescence changes of TPE-Gal $2(20 \mu \mathrm{M})$ or TPE-Gal $4(20 \mu \mathrm{M})$ in PBS solution ( $\left.\mathrm{pH} 7.4,0.01 \mathrm{M}, \lambda_{\mathrm{ex}}=320 \mathrm{~nm}\right)$ at $25^{\circ} \mathrm{C}$ with addition of DMB1 $(0-30 \mu \mathrm{M})$ and DMB2 $(0-80 \mu \mathrm{M})$. tensity of DMB1 remained unchanged (Figure 4). The PET effect from the arylboronate moiety might be influenced by the assembly with TPE-glycocluster, leading to a partial recovery of the fluorescence emission of DMB2. On the contrary, while the ICT effect from the boronate moiety still quenches its fluorescence, DMB1 has also proven to accept the energy but not released through irradiative process, thus only quenching the fluorescence of TPE-glycoclusters.

Then, we measured the color and fluorescence change of glyco-dots upon addition of peroxynitrite in PBS buffer. We observed a clear change in solution color of the self-assembled TPE-glycodots (Figure S2) after being exposed to peroxynitrite in PBS buffer. Besides, we measured the fluorescence ratiometric change of the glyco-dots in the presence of peroxynitrite in pure PBS buffer. Similarly, we observed a gradually quenched TPE fluorescence upon titration with both DM probes. However, unlike DMB1 alone, the reaction product of DMB1 and peroxynitrite (i.e. DM-OH) exhibited a fluorescence enhancement, indicative of a FRET process $\left(\Phi_{\mathrm{ET}}=89 \%\right)$ from TPE to DM-OH (Figure 5). Interestingly, the fluorescence intensity at $625 \mathrm{~nm}$ of DM-OH with TPE-Gal ${ }_{2}$ was much stronger than that with TPE$\mathrm{Gal}_{4}\left(\Phi_{\mathrm{ET}}=59 \%\right)$, which suggests that, in the glyco-dot system, the distance between the disubstituted TPE and DM-OH was closer for achieving a higher FRET efficiency than the tetra-substituted counterpart. A similar phenomenon was observed for the DMB2-based glyco-dots. The single fluorescence peak and intense emission at $625 \mathrm{~nm}$ indicated of high energy transfer $\left(\Phi_{\mathrm{ET}}=90 \%\right)$ between TPE-Gal ${ }_{2}$ and DM-OH in the presence of peroxynitrite. However, the characteristic double emission peaks of DMB2 at $590 \mathrm{~nm}$ overlapped with the emission of $\mathrm{DM}-\mathrm{OH}$ at $625 \mathrm{~nm}$ in TPE-Gal ${ }_{4}$ participation (Figure 5), thereby was similar to the results without peroxynitrite (Figure 4). The self-assembly affinity of TPE-Gal ${ }_{4}$ with DM probes in the presence of peroxynitrite is still unknown and worthy of investigation.

To further examine that TPE-glycocluster could assemble with DM-OH in order to block its ACQ effect, TPE-Gal ${ }_{2}$ or TPE-
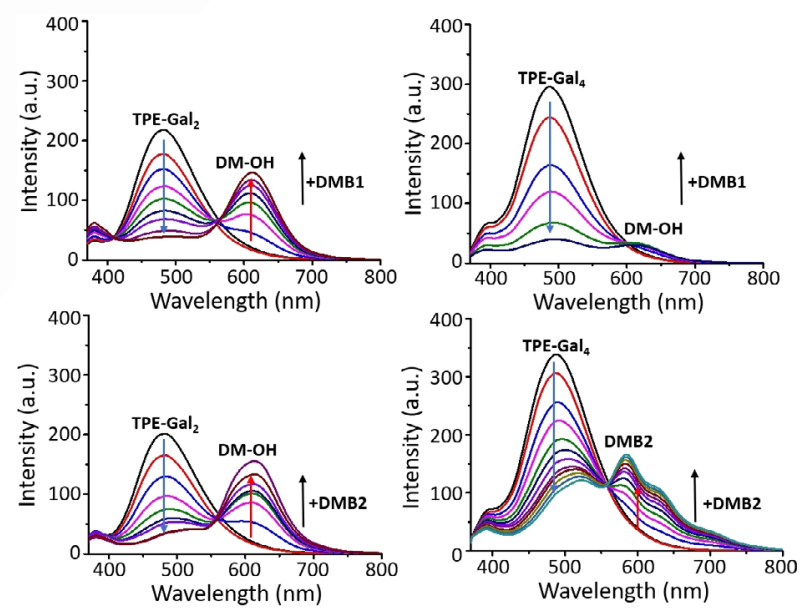

Figure 5. Fluorescence changes of TPE-Gal $2(20 \mu \mathrm{M})$ or TPE-Gal ${ }_{4}(20 \mu \mathrm{M})$ in PBS solution ( $\left.\mathrm{pH} 7.4,0.01 \mathrm{M}, \lambda_{\mathrm{ex}}=320 \mathrm{~nm}\right)$ at $25^{\circ} \mathrm{C}$ premixed with excess peroxynitrite $(100 \mu \mathrm{M})$, followed by addition of DMB1 $(0-30 \mu \mathrm{M})$ and DMB2 $(0-50 \mu \mathrm{M})$ 
$\mathrm{Gal}_{4}$ was added into the PBS solution of DMB1 or DMB2 with excessive peroxynitrite. Using the DM excitation of $470 \mathrm{~nm}$, we observed a gradually enhanced fluorescence of both DM probes in pure PBS solution (Figure 6). After self-assembly with TPE-Gal ${ }_{2}$ or TPE-Gal ${ }_{4}$, DMB1, respectively, displayed 11.5 -fold ( $\Phi 0.02-0.23$ ) or 15.5 -fold ( $\Phi 0.02$ to 0.31 ) fluorescence increase in the presence of peroxynitrite. Slightly weaker fluorescence signal were increased by DMB2-based glyco-dots self-assembled with TPE-Gal ${ }_{2}\left(10.5\right.$-fold, $\Phi$ 0.02-0.21) or TPE-Gal ${ }_{4}$ (12-fold, $\Phi 0.02$ to 0.24). Meanwhile, other TPE-based glycoclusters (Glc, Man, Fuc) could increase the fluorescence intensity of DM probes after self-assembly, similar to TPE-Gal ${ }_{2}$ or TPE$\mathrm{Gal}_{4}$ (Figure S5). These results clearly suggest that the self-assembly with TPE-glycoclusters largely enhance the fluorescence intensity of the relatively hydrophobic DM probes for peroxynitrite in pure PBS buffer.
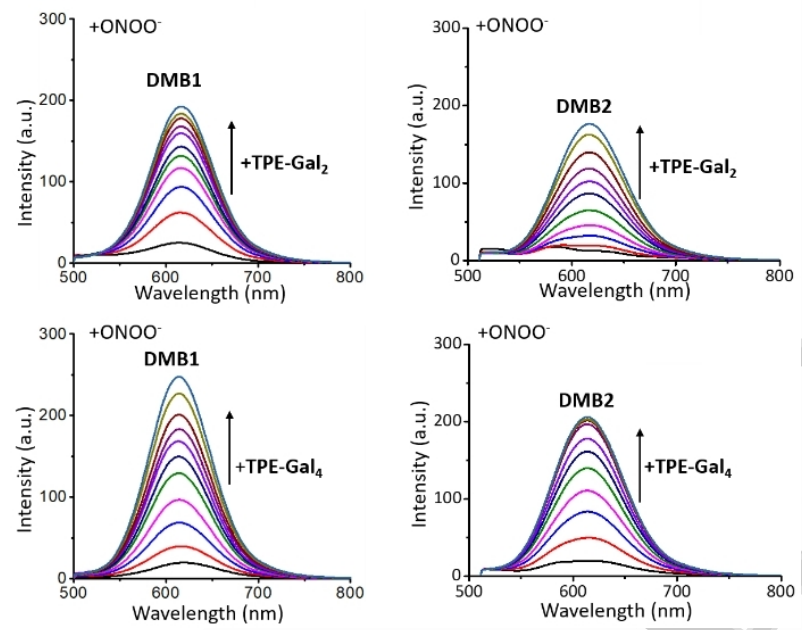

Figure 6. Fluorescence changes of DMB1 $(10 \mu \mathrm{M})$ and DMB2 $(10 \mu \mathrm{M})$ premixed with peroxynitrite $(100 \mu \mathrm{M})$ in PBS buffer $(\mathrm{pH} 7.4,0.01 \mathrm{M}$, $\left.\lambda_{\mathrm{ex}}=470 \mathrm{~nm}\right)$ at $25^{\circ} \mathrm{C}$, followed by addition of TPE-Gal $2(0-200 \mu \mathrm{M})$ and TPE-Gal $4(0-200 \mu \mathrm{M})$.

\section{Fluorescence properties of the glyco-dots}

To evaluate the response of glyco-dots to $\mathrm{ONOO}^{-}$, some fundamental parameters were measured in full PBS buffer (without additional $20 \%$ of $\mathrm{MeCN}$ ). We firstly analyzed the time-dependent fluorescence changes of glyco-dots in presence of peroxynitrite $(150 \mu \mathrm{M})$. Both probes exhibited a fluorescence "turn-on" response to peroxynitrite in PBS buffer (Figure 7A, Figure S6). An excess of peroxynitrite was used to ensure that all probe molecules react producing the fluorescent species DM-OH. An clear fluorescence enhancement was observed for the probes in the presence of peroxynitrite and this change was time dependent. DMB1-based glyco-dots reacted faster than the DMB2-based one. This could be due to the assumption that DMB1 produces DM-OH by a single "synthetic" step while DMB2 requires a two-step reaction for the elimination of quinone methide. The fluorescence enhancement capacity of TPE-Gal ${ }_{4}$ based glyco-dots seemed to be stronger than that of the TPE-Gal ${ }_{2}$ based counterparts.
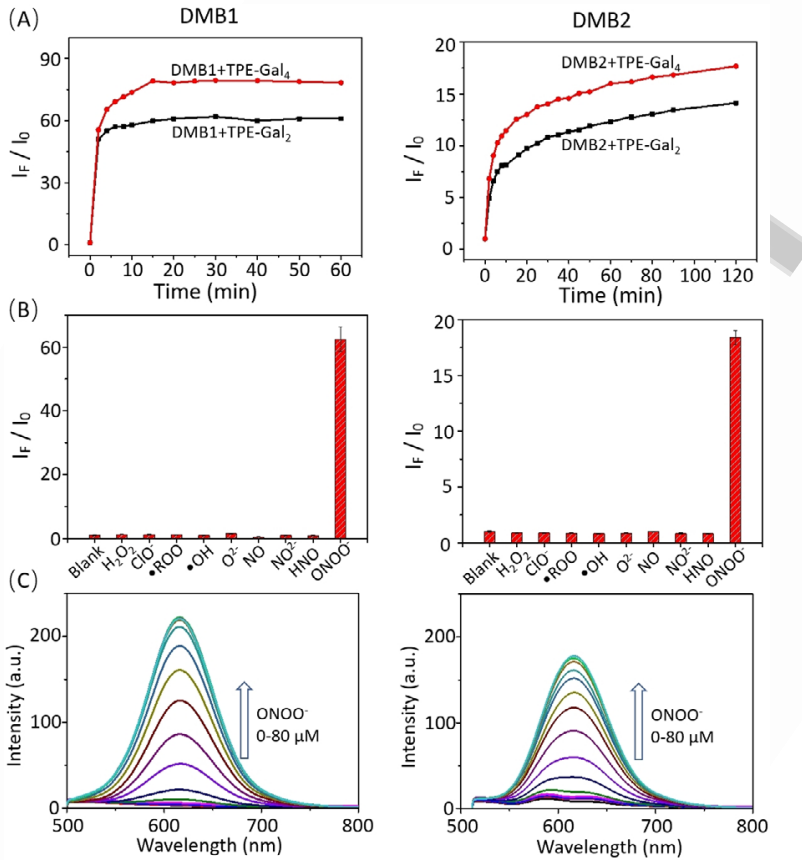

Figure 7. (A) Time-dependent fluorescence enhancement of these glyco-dots for $\mathrm{ONOO}^{-}$response. (B) Selectivity of two glyco-dots self-assembled from DM probes and TPE-Gal ${ }_{4}$ for several ROS, RNS and peroxynitrite. (C) Fluorescence emission enhancements of two glyco-dots self-assembled with DM probes and TPE-Gal ${ }_{4}$ with addition of peroxynitrite concentration $(0-80 \mu \mathrm{M})$. The glyco-dots were self-assembled from DM probes $(10 \mu \mathrm{M})$ and TPE-based glycoclusters $(150 \mu \mathrm{M})$, and then detect the ROS, RNS or peroxynitrite $(150 \mu \mathrm{m})$ in PBS buffer $\left(\mathrm{pH} 7.4,0.01 \mathrm{M}, \lambda_{\mathrm{ex}}=470 \mathrm{~nm}\right)$ at $25^{\circ} \mathrm{C}$.

Then, we tested the selectivity of the four glyco-dots for peroxynitrite in the presence of several potential interfering ROS and RNS (Figure 7B, Figure S7). All glyco-dots exhibited a strong emission intensity enhancement at $625 \mathrm{~nm}$ upon addition of peroxynitrite, whereas minimal fluorescence changes were observed in the presence of other ROS and RNS. The association of the DM probes with TPE-glycoclusters did not compromise the selectivity of the sensing system. We also measured the fluorescence intensity changes of the glyco-dots with increasing peroxynitrite in PBS (Figure 7C, Figure S8). All glyco-dots exhibited an obvious fluorescence enhancement with addition of peroxynitrite $(0-80 \mu \mathrm{M})$ in pure PBS buffer. Among them, the glyco-dots composed of DMB1 showed a higher fluorescence increase than those composed of DMB2, which could be similarly ascribed to the single "synthetic" step process of the former. TPE-Gal ${ }_{4}$ better improved the fluorescence properties of the glyco-dots comparing to the TPE-Gal system, thus suggesting its ability to better overcome the ACQ problem of DM. Sigmoidal titration curves were obtained for the glyco-dots with increasing peroxynitrite; however DM probes alone showed minimal fluorescent response to the highest concentration of peroxynitrite in PBS buffer.

\section{Glyco-dots imaging of peroxynitrite in living cells}

Human hepatoma cell line (HepG2) highly expresses the asialoglycoprotein receptor (ASPGr) which binds preferentially to 
galactosides. Hence, the glyco-dots self-assembly obtained from DM probes and galactose-based glycoclusters were incubated with HepG2 cells and employed to detect the exogenous and endogenous $\mathrm{ONOO}^{-}$.

We first determined that DMB1 showed a twofold higher fluorescence intensity in cells treated with SIN-1 (a peroxynitrite inducer) than DMB2 alone (Figure 8). In contrast, the fluorescence intensity of the glyco-dots produced in the cells was observed to be much stronger than that of the probes alone. We observed that: 1) TPE-Gal ${ }_{4}$ led to a better fluorescence enhancement for both DM probes than TPE-Gal ${ }_{2}$, and that 2) DMB1-based glyco-dots performed better than DMB2-based glyco-dots, similar to the observation in the solution-state fluorescence studies.

To test whether the enhanced fluorescence imaging effect would be the result of galactose-ASGPr interaction facilitating active endocytosis of the material, the glyco-dots formed between DMB1 and TPE-based glycoclusters modified with other carbohydrate epitopes (Glc, Man and Fuc) were used. To our surprise, all these glycoclusters enhanced the fluorescence imaging effect of DMB1 in HepG2 cells (Figure S9). This observation differs from our previous study using PDI-based glycoclusters for targeted delivery of a DM-based imaging agent to cancer cells that highly express specific carbohydrate receptors. ${ }^{[13]}$ This previous study indicated that PDI-based glyco-dots could target selectively HepG2 cells based on a carbohydratereceptor interaction at the cell surface. We preliminarily hypothesized that, unlike PDI-based glyco-dots ${ }^{[13]}$ that predominantly facilitate multivalent carbohydrate-receptor interactions, the TPE-based glyco-dots might adopt a different mode of interaction with the cell membrane. The good water solubility of TPE-based glyco-dots may improve the dispersion of DM probes in aqueous solution that is beneficial for cell endocytosis and TPE-based glyco-dots may increase the fluidity of the

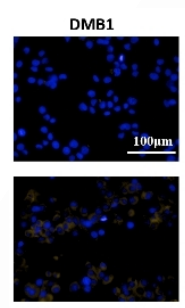

DMB2

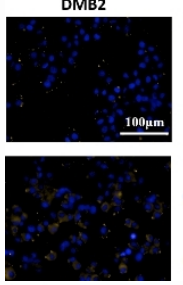

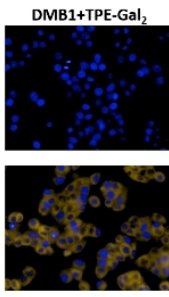

DMB2+TPE-Gal,

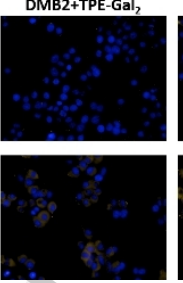

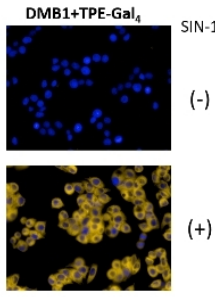

DMB2+TPE-Gal

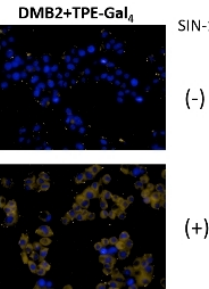

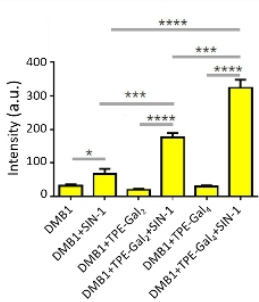

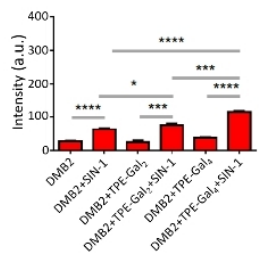

Figure 8. Fluorescence imaging and quantification of HepG2 cells incubated with glyco-dots self-assembled from DM probes (10 $\mu \mathrm{M}$, yellow) and TPEbased glycoclusters $(150 \mu \mathrm{M})$ without $(-)$ or with $(+)$ a subsequent addition of SIN-1 (150 $\mu \mathrm{M}$, a peroxynitrite inducer). Excitation and emission wavelengths for glyco-dots are $470-490 \mathrm{~nm}$ and $580-650 \mathrm{~nm}$, respectively. The cell nuclei were stained by Hoechst 33342 (blue). Error bars represent the s.d. ${ }^{*} p<0.05,{ }^{* *} p<0.01,{ }^{* * *} p<0.001,{ }^{* * * *} p<0.0001 ; n=3$. cell membrane, thus improving the penetration of the DM probes into cells. physical assays are needed to confirm this hypothesis, which is not the main focus of the present study that develops a sensitivity-enhanced system by means of the simple supramolecular self-assembly between fluorogenic probes and glycoclusters.

To explore where the probes located in cells, the colocalization experiments was performed to confirm the subcellular distribution of glyco-dots (DMB1+TPE-Gal ${ }_{4}$ ) in HepG2 cells (Figure 9). The fluorescence of DMB1 almost completely overlapped with the signal from a commercial MitoTracker Red for mitochondrial target. However, the fluorescence of DMB1 did not coincide with the fluorescence of LysoTracker, a commercial lysosome marker. The fluorescence intensity of the linear region of interest (ROls) of two channels across a selected cell altered close synchrony, and the Pearson's colocalization coefficient were determined to be 0.92 for mitochondria and 0.45 for lysosome, respectively. Therefore, the glyco-dots have ability to accumulate and image in mitochondria for $\mathrm{ONOO}^{-}$generation and functions.

We also tested the response of the glyco-dots for endogenous peroxynitrite produced by lipopolysaccharide (LPS) in another macrophage cell line (RAW264.7) (Figure 10). Similarly, we observed an enhanced fluorescence intensity for the glycodots after reacting with endogenous peroxynitrite. This suggests that our self-assembly strategy was generally applicable to enhancing the imaging capacity of otherwise cell imperme-

(A)
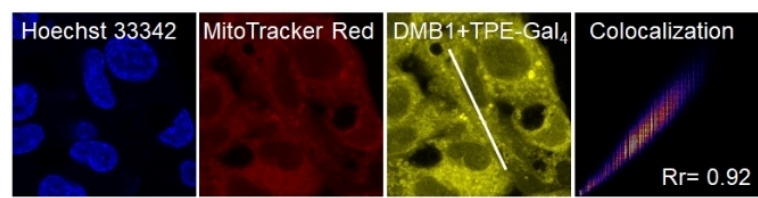

(B)
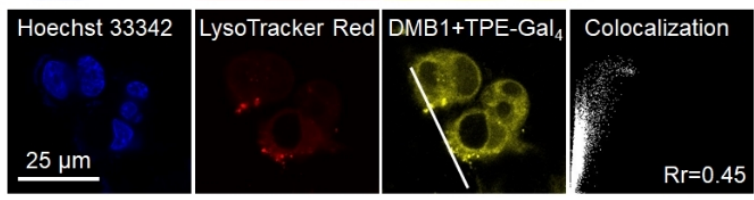

(C)
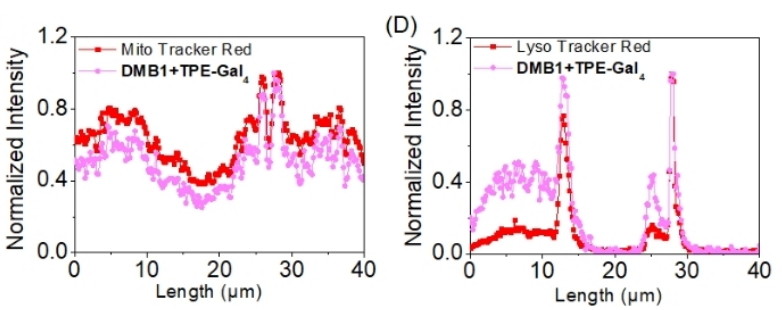

Figure 9. Fluorescence co-localization of DMB1 + TPE-Gal ${ }_{4}$ with (A) MitoTracker Red (a mitochondria tracker, $500 \mathrm{nM}$ ) and (B) LysoTracker Red (a lysosome tracker, $500 \mathrm{~nm}$ ) in HepG2 cells. (C) Fluorescence quantification of selected cellular regions (white solid lines in panel A) of DMB1 + TPE-Gal ${ }_{4}$ and MitoTracker Red (500 nM) in HepG2 cells. (D) Fluorescence quantification of selected cellular regions (white solid lines in panel B) of DMB1 + TPE-Gal and LysoTracker Deep Red (500 nM) in HepG2 cells. SIN-1 (500 $\mu \mathrm{M})$ was then added to activate fluorescent. Excitation wavelengths for DMB1 + TPE-Gal and subcellular organelle dye (LysoTracker Red or MitoTracker Red) are $488 \mathrm{~nm}$ and $559 \mathrm{~nm}$, respectively. Emission wavelengths for DMB1 + TPE$\mathrm{Gal}_{4}$ and subcellular organelle dye (LysoTracker Red or MitoTracker Red) are 580-650 $\mathrm{nm}$ and 570-590 $\mathrm{nm}$, respectively. 

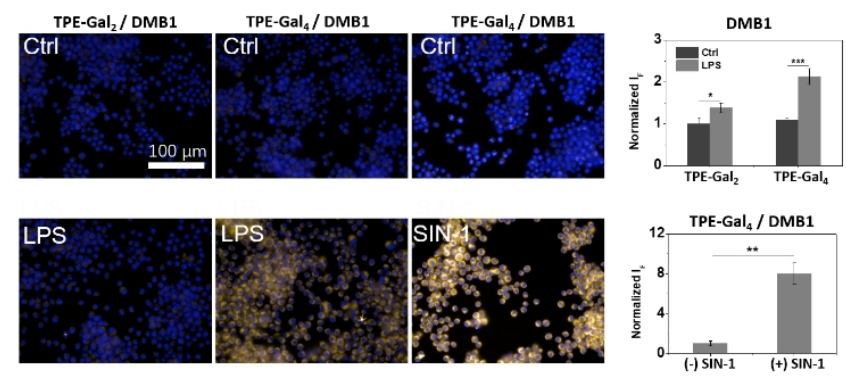

Figure 10. Fluorescence imaging and quantification of RAW 264.7 incubated with glyco-dots self-assembled from DM probes (10 $\mu \mathrm{M}$, yellow) and TPEbased glycoclusters $(150 \mu \mathrm{M})$ without or with a subsequent addition of lipopolysaccharide (LPS, $\left.1 \mathrm{mg} \mathrm{mL}^{-1}\right)$ or SIN-1 $(150 \mu \mathrm{M})$. Excitation and emission wavelengths for glyco-dots are $470-490 \mathrm{~nm}$ and $580-650 \mathrm{~nm}$, respectively. The cell nuclei were stained by Hoechst 33342 (blue). Error bars represent the s.d. ${ }^{*} p<0.05,{ }^{* *} p<0.01,{ }^{* * *} p<0.001 . n=3$.

able fluorogenic probes in different cells. The glyco-dots were also shown to be nontoxic to HepG2 cells with concentrations higher than that use for fluorescence imaging (Figure S10).

\section{Conclusions}

Two red-emitting $(625 \mathrm{~nm})$ phenylboronate fluorescent probes based on dicyanomethylene-4H-pyran (DMB1 and DMB2) have been designed and synthesized. Their sensing mechanism for peroxynitrite was studied by MS and HPLC. However, an insufficient fluorescence response was observed after exposure to peroxynitrite in full PBS buffer, which we ascribed to the ACQ effect. The poor water solubility and ACQ effect observed prompted our attempt of the supramolecular self-assembly between water soluble TPE-based glycoclusters and DM probes. The resulting glyco-dots displayed a significantly improved sensitivity $(\approx 60$-fold) for peroxynitrite in pure PBS buffer. The glyco-dots also exhibited a better fluorescence imaging effect for intracellular peroxynitrite (exogenously added and LPSstimulated) than the probes alone, thereby offering scope for the development of other similar self-assembling systems for chemical biology studies.

\section{Experimental Section}

\section{Synthesis of probes}

Detailed descriptions of the syntheses can be found in the Supporting Information.

\section{UV/Vis absorbance spectroscopy}

The UV/Vis absorbance measurements were carried out at room temperature using a Varian Cary 60 UV/Vis spectrophotometer. All spectra were corrected for background intensities by subtracting the spectra of pure solvent measured under identical conditions. UV/Vis/NIR absorption spectra $(0-800 \mathrm{~nm})$ of probes.

\section{Fluorescence spectroscopy}

The fluorescence measurements were carried out at room temperature using an Agilent Cary Eclipse fluorescence spectrophotometer.

\section{Fluorescence quantum yields}

DMB1 or DMB2 (with a final concentration of $30 \mu \mathrm{M}$ ) was dissolved in aqueous $0.01 \mathrm{M}$ PBS (pH 7.4). DMB1 and DMB2 (with a final concentration of $10 \mu \mathrm{M}$ ) with addition of peroxynitrite (with a final concentration of $100 \mu \mathrm{m})$ dissolved in aqueous $0.01 \mathrm{M}$ PBS (pH 7.4). Rhodamine $B$ (with a final concentration of $5 \mu \mathrm{M}$ ) was dissolved in aqueous $0.01 \mathrm{M}$ PBS ( $\mathrm{pH} 7.4$ ). The UV/Vis absorbance measurements were carried out at room temperature using a Varian Cary 60 UV/Vis spectrophotometer. UV absorption values were collected at $470 \mathrm{~nm}$. The fluorescence measurements were carried out at room temperature by an Agilent Cary Eclipse fluorescence spectrophotometer with an excitation wavelength of $480 \mathrm{~nm}$. Integrated fluorescence curve between $500 \mathrm{~nm}$ and $850 \mathrm{~nm}$. Finally, the fluorescence quantum yield was calculated according to Equation (1):

$$
Y_{u}=Y_{r} \cdot \frac{F_{u}}{F_{r}} \cdot \frac{A_{r}}{A_{u}} \cdot \frac{n_{u}^{2}}{n_{r}^{2}}
$$

$\mathrm{u}=$ probe, $\mathrm{r}=$ rhodamine $\mathrm{B}, Y=$ fluorescence quantum yield, $F=$ integrated fluorescence intensity, $A=$ absorbance, $n=$ refractive index.

\section{Supramolecular self-assembly of glyco-dots}

DM probe (1 $\mathrm{mM}, \mathrm{DMSO}$ ) was added to a solution of TPE-based glycoclusters ( $15 \mathrm{~mm}$, PBS buffer). The resulting mixture was stirred during $30 \mathrm{~min}$ to produce the supramolecular glyco-dots for subsequent experiments.

\section{Preparation of ROS/RNS}

Details descriptions of the ROS and RNS preparation can be found in the Supporting Information.

\section{Transmission electron microscope (TEM)}

A droplet of probes (DMB1 and DMB2 $=10 \mu \mathrm{M}$, TPE$^{-G a l_{2}}$ and TPE$\mathrm{Gal}_{4}=150 \mu \mathrm{M}$ ) was dropped onto 200 mesh holey carbon copper grids for TEM characterizations. JEOL 1400 equipped with a Gatan Orius charged-coupled device camera and Tridiem energy filter operating at $200 \mathrm{kV}$ was used for TEM, and data were processed using Image J software.

\section{Dynamic light scattering}

Dynamic light scattering carried out using a Horiba LB-550 Dynamic Light Scattering Nano-Analyzer.

\section{Statistical analysis}

Error bars represent s.d. Data analysis was performed with GraphPad PRISM (GraphPad Software, Inc.) using two-tailed unpaired Student's t-test. $P$-values $<0.05$ were considered statistically significant. All experiments were repeated at least three times with representative data shown. 


\section{Cell culture}

HepG2 and RAW264.7 cells were maintained in a Dulbecco's Modified Eagle's Medium (Invitrogen, Carlsbad, CA, USA) supplemented with $10 \%$ fetal bovine serum (Gibco, Gland Island, NY, USA) in a humidified atmosphere of $5 \% \mathrm{CO}_{2}$ and $95 \%$ air at $37^{\circ} \mathrm{C}$ and split when the cells reached $90 \%$ confluency.

\section{Fluorescence imaging of cells}

Cells were seeded on a black 96-well microplate with optically clear bottom (Greiner bio-one, Germany), overnight. To produce peroxynitrite exogenously, the cells were first incubated with DM probe/ TPE-glycocluster (10 $\mu \mathrm{m} / 150 \mu \mathrm{M}, 1 \%$ DMSO in PBS, pH 7.4) for $30 \mathrm{~min}$, followed by incubation with SIN-1 $(150 \mu \mathrm{M})$ for $30 \mathrm{~min}$. The cell nuclei were stained with Hoechst $33342\left(5 \mu \mathrm{g} \mathrm{mL}^{-1}\right)$ at $37^{\circ} \mathrm{C}$ in a humidified atmosphere of $5 \% \mathrm{CO}_{2}$ in air for $5 \mathrm{~min}$. Then, cells were washed with PBS (phosphate buffered saline) three times. The fluorescence images were recorded using an Operetta high-content imaging system (PerkinElmer, US) and quantified and plotted by Columbus analysis system (PerkinElmer, US).

\section{Cell viability assay}

Cells were plated, overnight, on 96-well plates in growth medium. After seeding, cells were treated with glycodots of different concentrations for 48 hours. Then, $10 \mu \mathrm{L}$ per well of MTS/PMS (20:1, Promega Corp) solution was added to each well containing $100 \mu \mathrm{L}$ of growth medium. After incubation at $37^{\circ} \mathrm{C}$ under $5 \% \mathrm{CO}_{2}$ for $2 \mathrm{~h}$, the absorbance of the solutions was measured at $470 / 480 \mathrm{~nm}$, using an M5 microplate reader (Molecular Device, USA). The optical density of the result in MTS assay was directly proportional to the number of viable cells.

\section{Acknowledgements}

The authors thank the financial support from Universite Claude Bernard Lyon 1 and CNRS, the Natural Science Foundation of China (nos. 21788102, 91853201, 21722801 and 21776078), the Shanghai Municipal Science and Technology Major Project (No. 2018SHZDZX03), the National Key Sci-Tech Special Projects of Infection Diseases of China (2018ZX10732202), and the international cooperation program of Shanghai Science and Technology Committee (17520750100). Lei Dong is grateful to the China Scholarship Council for a PhD stipend (201606740066).

\section{Conflict of interest}

The authors declare no conflict of interest.

Keywords: cell imaging • glycoclusters • peroxynitrite
detection - self-assembly - tetraphenylethylene

Manuscript received: June 8,2020

Accepted manuscript online: June 17, 2020

Version of record online: $\mathbf{\square} \mathbf{\square}, 0000$

[1] a) K.-T. Lin, J.-Y. Xue, M. C. Lin, E. G. Spokas, F. F. Sun, P. Y.-K. Wong, Am. J. Physiol. Cell Physiol. 1998, 274, C855-C860; b) K. B. Li, L. Dong, S. Zhang, W. Shi, W. P. Jia, D. M. Han, Talanta 2017, 165, 593-597.

[2] P. Pacher, J. S. Beckman, L. Liaudet, Physiol. Rev. 2007, 87, 315-424.

[3] a) J. Zhang, X. Zhen, J. Zeng, K. Pu, Anal. Chem. 2018, 90, 9301-9307; b) X. Xie, F. Tang, G. Liu, Y. Li, X. Su, X. Jiao, X. Wang, B. Tang, Anal. Chem. 2018, 90, 11629-11635; c) Y. Wu, A. Shi, Y. Li, H. Zeng, X. Chen, J. Wu, X. Fan, Analyst 2018, 143, 5512-5519; d) L. Li, M. Zan, X. Qie, J. Yue, P. Miao, M. Ge, Z. Chang, Z. Wang, F. Q. Bai, H. X. Zhang, J. K. Ferri, W. F. Dong, J. Phys. Chem. A 2018, 122, 217-223; e) Y. Li, X. Xie, X. Yang, M. Li, X. Jiao, Y. Sun, X. Wang, B. Tang, Chem. Sci. 2017, 8, 4006-4011; f) K. Debowska, D. Debski, B. Michalowski, A. Dybala-Defratyka, T. Wojcik, R. Michalski, M. Jakubowska, A. Selmi, R. Smulik, L. Piotrowski, J. Adamus, A. Marcinek, S. Chlopicki, A. Sikora, Chem. Res. Toxicol. 2016, $29,735-746$.

[4] A. Sikora, J. Zielonka, M. Lopez, J. Joseph, B. Kalyanaraman, Free Rad. Biol. Med. 2009, 47, $1401-1407$.

[5] a) M. Weber, A. B. Mackenzie, S. D. Bull, T. D. James, Anal. Chem. 2018, 90, 10621 -10627; b) J. Zhou, Y. Li, J. Shen, Q. Li, R. Wang, Y. Xu, X. Qian, RSC Adv. 2014, 4, 51589-51592; c) J. Kim, J. Park, H. Lee, Y. Choi, Y. Kim, Chem. Commun. 2014, 50, 9353-9356; d) F. Yu, P. Song, P. Li, B. Wang, K. Han, Analyst 2012, 137, 3740-3749.

[6] A. C. Sedgwick, H. H. Han, J. E. Gardiner, S. D. Bull, X. P. He, T. D. James, Chem. Commun. 2017, 53, 12822-12825.

[7] X. Sun, Q. Xu, G. Kim, S. E. Flower, J. P. Lowe, J. Yoon, J. S. Fossey, X. Qian, S. D. Bull, T. D. James, Chem. Sci. 2014, 5, 3368.

[8] a) A. A. Rajkiewicz, K. Skowerski, B. Trzaskowski, A. Kajetanowicz, K. Grela, ACS Omega 2019, 4, 1831-1837; b) A. Angelova, M. Drechsler, V. M. Garamus, B. Angelov, ACS Omega 2018, 3, 3235-3247.

[9] a) X.-P. He, R.-H. Li, S. Maisonneuve, Y. Ruan, G.-R. Chen, J. Xie, Chem. Commun. 2014, 50, 14141-14144; b) K.-B. Li, D. Zhou, X.-P. He, G.-R. Chen, Dyes Pigm. 2015, 116, 52-57; c) L. Dong, Y. Zang, D. Zhou, X. P. He, G. R. Chen, T. D. James, J. Li, Chem. Commun. 2015, 51, $11852-$ 11855 ; d) K. B. Li, N. Li, Y. Zang, G. R. Chen, J. Li, T. D. James, X. P. He, H. Tian, Chem. Sci. 2016, 7, 6325-6329.

[10] a) D. K. Ji, G. R. Chen, X. P. He, H. Tian, Adv. Funct. Mater. 2015, 25, 3483-3487; b) D. K. Ji, Y. Zhang, Y. Zang, J. Li, G. R. Chen, X. P. He, H. Tian, Adv. Mater. 2016, 28, 9356-9363.

[11] a) K. Gu, W. Qiu, Z. Guo, C. Yan, S. Zhu, D. Yao, P. Shi, H. Tian, W. H. Zhu, Chem. Sci. 2019, 10, 398-405; b) K. Gu, Y. Xu, H. Li, Z. Guo, S. Zhu, S. Zhu, P. Shi, T. D. James, H. Tian, W. H. Zhu, J. Am. Chem. Soc. 2016, 138, 5334-5340.

[12] M. Donnier-Maréchal, N. Galanos, T. Grandjean, Y. Pascal, D. K. Ji, L. Dong, E. Gillon, X. P. He, A. Imberty, E. Kipnis, R. Dessein, S. Vidal, Org. Biomol. Chem. 2017, 15, 10037-10043.

[13] Y. Liu, D. K. Ji, L. Dong, N. Galanos, Y. Zang, J. Li, S. Vidal, X. P. He, Chem. Commun. 2017, 53, 11937-11940.

[14] M. Donnier-Maréchal, S. Abdullayev, M. Bauduin, Y. Pascal, M. Q. Fu, X. P. He, E. Gillon, A. Imberty, E. Kipnis, R. Dessein, S. Vidal, Org. Biomol. Chem. 2018, 16, 8804-8809.

[15] G. Zhao, Y. Zhu, S. Guang, F. Ke, H. Xu, New J. Chem. 2018, 42, 555-563.

[16] H.-T. Feng, J.-B. Xiong, Y.-S. Zheng, B. Pan, C. Zhang, L. Wang, Y. Xie, Chem. Mater. 2015, 27, 7812-7819. 


\section{FULL PAPER}

Self-assembly of TPE-based glycoclusters with fluorogenic probes allowed for the detection of peroxynitrite in vitro and in cell assays through the recovery of proper water solubility and fluorescence emission.

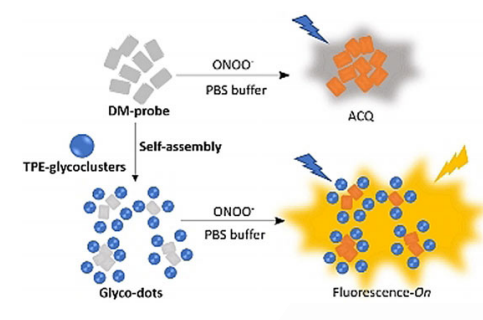

Chemical Biology

L. Dong, M. Fu, L. Liu, H.-H. Han, Y. Zang, G.-R. Chen, J. Li, ${ }^{*}$ X.-P. He, ${ }^{*}$ S. Vidal*

\section{घ-口}

Supramolecular Assembly of TPEBased Glycoclusters with Dicyanomethylene-4H-pyran (DM) Fluorescent Probes Improve Their Properties for Peroxynitrite Sensing and Cell Imaging

\section{@UnivLyon1 SPACE RESERVED FOR IMAGE AND LINK}

Share your work on social media! Chemistry - A European Journal has added Twitter as a means to promote your article. Twitter is an online microblogging service that enables its users to send and read text-based messages of up to 140 characters, known as "tweets". Please check the pre-written tweet in the galley proofs for accuracy. Should you or your institute have a Twitter account, please let us know the appropriate username (i.e., @accountname), and we will do our best to include this information in the tweet. This tweet will be posted to the journal's Twitter account @ChemEurJ (follow us!) upon online publication of your article, and we recommended you to repost ("retweet") it to alert other researchers about your publication.

Please check that the ORCID identifiers listed below are correct. We encourage all authors to provide an ORCID identifier for each coauthor. ORCID is a registry that provides researchers with a unique digital identifier. Some funding agencies recommend or even require the inclusion of ORCID IDs in all published articles, and authors should consult their funding agency guidelines for details. Registration is easy and free; for further information, see http://orcid.org/.

Dr. Lei Dong

Mengqi Fu

Prof. Lifang Liu

Hai-Hao Han

Prof. Yi Zang

Prof. Guo-Rong Chen

Jia Li

Prof. Xiao-Peng $\mathrm{He}$

Dr. Sébastien Vidal http://orcid.org/0000-0001-7812-698X 